\title{
Emotional Resilience and Bariatric Surgical Teams: a Priority in the Pandemic
}

\author{
Yitka Graham ${ }^{1,2,3}$ (1) Kamal Mahawar ${ }^{1,2} \cdot$ Manel Riera $^{4} \cdot$ Omar Islam $^{2} \cdot$ Aparna Ghovil Bhasker $^{5} \cdot$ Michael Wilson $^{6}$. \\ Abd Tahrani $^{7,8,9} \cdot$ Violeta Moize $^{10} \cdot$ Angela Leal $^{11} \cdot$ Catherine Hayes $^{1}$
}

Received: 23 June 2020 / Revised: 16 October 2020 / Accepted: 21 October 2020 / Published online: 2 November 2020

(C) Springer Science+Business Media, LLC, part of Springer Nature 2020

\begin{abstract}
The infection control measures implemented as a result of COVID-19 led to a postponement of bariatric surgical procedures across many countries worldwide. Many bariatric surgical teams were in essence left without a profession, with many redeployed to other areas of clinical care and were not able to provide the levels of patient support given before COVID-19. As the pandemic continues, some restrictions have been lifted, with staff adjusting to new ways of working, incorporating challenging working conditions and dealing with continuing levels of stress. This article explores the concept of emotional labour, defined as 'inducing or suppressing feelings in order to perform one's work', and its application to multidisciplinary teams working within bariatric surgery, to offer insight into the mental health issues that may be affecting healthcare professionals working in this discipline.
\end{abstract}

Keywords Bariatric surgery $\cdot$ Mental health $\cdot$ Emotional labour $\cdot$ Compassion fatigue $\cdot$ Resilience

\section{Introduction}

Following the World Health Organisation's (WHO) declaration of coronavirus as a global pandemic [1], the International Federation for the Surgery of Obesity and Metabolic Disorders (IFSO) issued guidance recommending the postponement of bariatric surgical procedures to protect both healthcare professionals and patients, many of whom were at increased risk of developing COVID-19 owing to pre-existing conditions, e.g. obesity, diabetes and cardiovascular disease [2]. Additionally, day to day practice has altered in terms of

Yitka Graham

yitka.graham@sunderland.ac.uk

1 Helen McArdle Nursing and Care Research Institute, Faculty of Health Sciences and Wellbeing, University of Sunderland, Sunderland, UK

2 Department of General Surgery, Sunderland Royal Hospital, South Tyneside and Sunderland NHS Foundation Trust, Kayll Road, Sunderland, UK

3 Faculdad de Psicologia, Universidad de Anahuac, Mexico City, Mexico

4 Department of General Surgery, Royal Shrewsbury Hospital, The Shrewsbury and Telford Hospitals NHS Trust, Mytton Oak Road, Shrewsbury, UK

5 Apollo and Gleneagles Global Hospitals, Mumbai, India patient care, with remote consultations, and home working, which for many is a new concept [3]. The halting of bariatric surgery and the impact of COVID-19 has, for some bariatric surgical professionals, meant redeployment (assigned to work in another clinical area) or being furloughed [4]. Many healthcare providers implemented recruitment freezes; all of these may be a source of stress for bariatric surgical professionals who are used to working in a specialist clinical environment.

Early evidence from the current and previous pandemics such as severe acute respiratory syndrome (SARS) has shown

6 Department of General Surgery, Forth Valley Royal Hospital, NHS Forth Valley, Stirling Road, Larbert, UK

7 Institute of Metabolism and Systems Research (IMSR), University of Birmingham, Birmingham, UK

8 Department of Diabetes and Endocrinology, University Hospitals Birmingham NHS Foundation Trust, Birmingham, UK

9 Centre of Endocrinology, Diabetes and Metabolism (CEDAM), Birmingham Health Partners, Birmingham, UK

10 Obesity Unit, Institut d'Investigacions Biomediques August Pi Sunyer (DIPABS), Centro de Investigacion Biomedica en Red de Diabetes y Enfermedades Metabolicas Asociadas (CIBERDEM), Hospital Clinic Barcelona, Barcelona, Spain

11 Department of Bariatric Surgery, Christus Muguerza Conchita Hospital, Monterrey, Mexico 
that healthcare professionals are at risk of adverse psychological effects in addition to physical ones [5]. Conditions such as depression, insomnia and anxiety, which may exist in all surgical disciplines in everyday practice [6], may be increased in the current pandemic $[7,8]$.

The psychological impact of SARS in a Taiwanese study of 1257 healthcare workers reported differences in mental health across two phases of the outbreak. In the initial phase (rapid spread of virus), $81 \%$ experienced anxiety and excessive worry, $68 \%$ experienced depression and associated negative impact on family relationships, $62 \%$ experienced somatic symptoms and $50 \%$ experienced sleep problems, underpinned by perceived threats to life, vulnerability amidst a high level of uncertainty. In the repair phase (infection brought under control), $83 \%$ experienced depression and poor family relationships, $78 \%$ experienced somatic symptoms, $77 \%$ experienced anxiety and excessive worrying and $54 \%$ experienced sleep problems. In the repair phase, there were higher rates of depression compared with higher levels of anxiety experienced in the initial phase. The reduction in anxiety was thought to be the result of the virus being under control and recognition that the disease was preventable. The high levels of depression were proposed to be a contributing factor for 120 nurses ( $8 \%$ of participants) tendering their resignations during the repair phase [9].

The first published study examining the mental health of clinical staff in Wuhan in the wake of the COVID-19 pandemic found that out of 994 staff, $37 \%$ had experienced subthreshold mental health incidents, $34 \%$ had mild disturbances, $22 \%$ had moderate issues and $6 \%$ had severe disturbances, with young women experiencing a higher burden of mental health issues compared with older females and males in the study cohort. In terms of support, $36 \%$ had accessed psychological materials (e.g. books), 50\% accessed media (e.g. text messages on self-help coping methods) and $17 \%$ had sought counselling or psychotherapy. There was little access to formal mental health service provision, but this was nevertheless perceived as an important resource [10]. Additional recommendations emphasised the importance of supporting the mental health and well-being of all frontline healthcare workers during the pandemic [11].

The WHO has recognised the impact of COVID-19 on mental health and well-being and has issued guidance on the provision of support specifically for health professionals in terms of being aware of feeling stressed, practicing self-care and encouraging staff to seek help for mental well-being if needed, and for healthcare organisations to have, and actively signpost to sources of support [12].

The emotional impact of the pandemic on the public in the USA with a representative sample of 1500 people over a 2week period in May was undertaken using the Pandemic Emotional Impact Scale (PEIS), consisting of a 16-question survey comparing levels of well-being pre-pandemic with the present. Findings showed that $55 \%$ of respondents reported more life stress than at the pre-pandemic level, $66 \%$ worried more about the health and safety of family and friends, $57 \%$ worried about their personal health and safety, $53 \%$ were more bored and 51\% reported feeling ill at ease or anxious [13].

Bariatric surgery is a unique specialty in that it relies on the skills and expertise of other healthcare professionals, such as psychologists, dietitians, nurses and pharmacists in addition to those of a surgeon pre-, peri- and post-operatively to maximise clinical outcomes. The skills of a surgeon are useless unless a patient understands, is prepared for and motivated to comply with the life-long changes that surgery brings, which requires a collaborative, multidisciplinary approach to patient care. This is what sets bariatric surgery apart from other surgical specialties.

\section{Emotional Labour}

Emotional labour is defined as managing or suppressing one's emotions in order to portray themselves in a socially constructed view of their profession, to produce a desired expectation in others [14]. Within the bariatric multidisciplinary team, the socially constructed norms of the roles of each team member have different attributes. As an example, the roles of surgeons and nurses are illuminated. The nature of the traditional surgeon-patient relationship invokes a need for trust, with the patient giving power and control to the surgeon in terms of intervention [15]; thus, there is an expectation for the surgeon to present these qualities. The nursing profession is inextricably linked with the concept of building a more compassionate, patient-centred culture, with robust evidence of this construction linked to positive patient outcomes [16]. For two professions involved in bariatric surgical multidisciplinary team working, a large degree of emotional labour is needed to fulfil the societal expectations of these roles. Additionally, bariatric patients are generally a complex and challenging cohort and suffer not only from the physical effects of obesity but the psychological issues, including many living with the stigma associated with obesity [17]. This also may place emotional burdens on professionals who provide care for these patients.

\section{Moral Injury}

Another way of looking at the mental well-being of bariatric healthcare teams is through the concept of moral injury. Defined as occurring when 'we perpetrate, bear witness to or fail to prevent an act that transgresses our deeply held moral beliefs' [18], such as knowing what patients need, but being unable to provide levels of care owing to external factors 
beyond individual control [18]. For bariatric surgical teams, the pandemic initially resulted in a global postponement of surgical procedures. Six months on, despite resumption of bariatric surgery in some countries, there are still increased waiting lists, altered or reduced working practices which affect the ability to provide the levels of patient contact and support at pre-pandemic levels. The ongoing and unpredictable nature of the pandemic means that the care provided to patients may not be what bariatric surgical teams are used to providing or that patients have come to expect. Many of the resources needed to provide these levels of care are out of both individual and the team's control, and the duality of wanting to support and provide optimal care and being unable to do so has the potential to perpetuate moral injury.

\section{Compassion Fatigue}

Like other healthcare professionals, bariatric multidisciplinary teams are exposed to the traumatic experiences of the patients they care for, which can lead to compassion fatigue, described as the "convergence of secondary traumatic stress and cumulative burnout, a state of physical and mental exhaustion caused by a depleted ability to cope with one's everyday environment' [19]. Compassion fatigue can adversely affect patient care, relationships with colleagues, and be a trigger for the development of mental health conditions, e.g. depression, anxiety and post-traumatic stress disorder. If not identified and addressed, this may ultimately lead to burnout [20].

With the pervading stigmatisation of obesity, the increased risks of contracting COVID-19 in the bariatric surgicalseeking population [21], the potential negative impact of confinement on patients' weight, worsening physical and mental health, slow restart of bariatric surgery and altered ways of working may also be triggers for compassion fatigue in bariatric surgical teams. The situation may be further exacerbated owing to the prolonged stress of the impact of COVID-19 in their everyday practice, the fears of contracting the virus and giving it to others, issues with personal protective equipment and witnessing the deaths of patients and colleagues [7].

\section{Recommendations}

The ongoing pandemic, and its continued impact on the provision of bariatric surgery, pre- and post-operative support, the need to be aware of emotional labour, moral injury and compassion fatigue, in terms of the potential impact on the mental health and well-being of bariatric surgical professionals is of paramount importance. Identifying and reflecting on the signs of these concepts, both in oneself and colleagues, mean being aware and acknowledging additional pressures in everyday practice and to integrate active mechanisms of coping and resilience.

As a result of the social distancing measures implemented in most organisations to contain the spread of COVID-19, remote consultations for surgical procedures, through telephone and videoconferencing, have been recommended [22], and their use has increased exponentially [23], especially in countries where this is not normal practice. In addition to protecting professionals and patients from infection, remote consultations offer opportunities for patient support and engagement and may contribute to reducing the current high levels of patients who do not attend post-surgical follow-up appointments [24]. A systematic review of studies examining eHealth pre- and post-operatively in bariatric surgical programmes found variation in study design, methodologies and method of eHealth delivery across the 38 studies. Although there was evidence of weight loss and other positive health outcomes, many of the studies were pilot and had acknowledged limitations [25]. The review was published prepandemic, which may not reflect the current situation and increased the use of eHealth technologies.

However, remote consultations have the potential to also support the mental health and well-being of bariatric surgical professionals, allowing them to continue to practice, albeit in a restricted capacity, and develop new and innovative ways of working, until such time that normal provision of bariatric surgery can resume.

A database of healthcare workers' experiences during the pandemic may be accessed at https://heroesresearch.org. Although not specific to bariatric surgery or any of the professional disciplines within bariatric surgical teams, HERO offers insights into other healthcare workers' narratives, which may provide a source of support through reading about others' experiences [26].

\section{Conclusion}

Unfortunately, the ongoing pandemic may mean bariatric multidisciplinary teams may face additional challenges. For example, patients are likely to resume their bariatric surgical journeys after many months on hold, often with little improvement or indeed worsening of both physical and mental health and lifestyle habits as a result of the impact of the pandemic. This may cause further difficulties at a time when additional pressure from other specialties and modern surgical prioritisation may affect the capacity to deliver the usual comprehensive bariatric surgical service.

The ability to be aware of, and understand emotional labour, moral injury and compassion fatigue within bariatric surgical teams, along with developing resilience strategies, is necessary to be able to adapt and work within this complex 
and difficult pandemic-induced environment and develop sustainable working practices for the future.

\section{Compliance with Ethical Standards}

Conflict of Interest Yitka Graham is a reviewer for Obesity Surgery. Kamal Mahawar is on the Editorial Board of Obesity Surgery. Omar Islam, Aparna Ghovil Bhasker, Michael Wilson, Abd Tahrani, Violeta Moize, Angela Leal and Catherine Hayes have no conflicts of interest.

Ethical Approval This article does not contain any studies with human participants or animals performed by any of the authors.

Informed Consent Does not apply.

\section{References}

1. World Health Organization. WHO Director-General's opening remarks at the media briefing on COVID-19 - 11 March 2020 Geneva: World Health Organisation; 2020. Available from: https://www.who.int/dg/speeches/detail/who-director-general-sopening-remarks-at-the-media-briefing-on-covid-19\%2D\%2D-11march-2020. Accessed 5 May 2020.

2. Yang W, Wang C, Shikora S, et al. Recommendations for metabolic and bariatric surgery during the COVID-19 pandemic from IFSO. Obes Surg. 2020;30:2071-3. https://doi.org/10.1007/ s11695-020-04578-1.

3. Pryor A. SAGES and EAES recommendations regarding surgical response to COVID-19 crisis Los Angeles: Society of American Gastrointestinal and Endoscopic Surgeons; 2020. Available from: https://www.sages.org/recommendations-surgical-response-covid$19 /$.

4. Hamilton JJ. Surgical, economic, and psychological impacts of SARS-COV-2 on a Kansas Community Hospital System. Am Surg. 2020;86(6):599-601. https://doi.org/10.1177/ 0003134820924394.

5. Adams JG, Walls RM. Supporting the health care workforce during the COVID-19 global epidemic. JAMA. 2020;323(15):1439-40. https://doi.org/10.1001/jama.2020.3972.

6. Gerada C, Jones R. Surgeons and mental illness: a hidden problem? BMJ. 2014;348:g2764. https://doi.org/10.1136/bmj.g2764.

7. Lai J, Ma S, Wang Y, et al. Factors associated with mental health outcomes among health care workers exposed to coronavirus disease 2019. JAMA Netw Open. 2020;3(3):e203976-e. https://doi. org/10.1001/jamanetworkopen.2020.3976.

8. Chang CS, Du PL, Huang IC. Nurses' perceptions of severe acute respiratory syndrome: relationship between commitment and intention to leave nursing. J Adv Nurs. 2006;54(2):171-9. https://doi. org/10.1111/j.1365-2648.2006.03796.x.

9. Chong M-Y, Wang W-C, Hsieh W-C, et al. Psychological impact of severe acute respiratory syndrome on health workers in a tertiary hospital. Br J Psychiatry. 2004;185(2):127-33. https://doi.org/10. 1192/bjp.185.2.127.

10. Kang L, Ma S, Chen M, et al. Impact on mental health and perceptions of psychological care among medical and nursing staff in Wuhan during the 2019 novel coronavirus disease outbreak: a cross-sectional study. Brain Behav Immun. 2020; https://doi.org/ 10.1016/j.bbi.2020.03.028.

11. Kang L, Li Y, Hu S, et al. The mental health of medical workers in Wuhan, China dealing with the 2019 novel coronavirus. Lancet Psychiatry. 2020;7(3):e14. https://doi.org/10.1016/s2215-0366(20) 30047-x.

12. World Health Organisation. Mental health and psychosocial considerations during the COVID-19 outbreak. Geneva: World Health Organisation; 2020.

13. Palsson O, Ballou S, Gray S. The U.S. National Pandemic Emotional Impact Report. Chapel Hill: University of North Carolina; 2020.

14. Hochschild AR. The managed heart: commercialisation of human feeling. 3rd ed. Oakland: University of California Press; 2012.

15. Axelrod DA, Goold SD. Maintaining trust in the surgeon-patient relationship: challenges for the new millennium. Arch Surg. 2000;135(1):55-61. https://doi.org/10.1001/archsurg.135.1.55.

16. Kinman G, Leggetter S. Emotional labour and wellbeing: what protects nurses? Healthcare. 2016;4(4):89. https://doi.org/10.3390/ healthcare4040089.

17. Puhl R, Himmelstein M, Pearl R. Weight stigma as a psychosocial contributor to obesity. Am Psychol. 2020;75(2):274-89. https://doi. org/10.1037/amp0000538.

18. Dean W, Talbot S, Dean A. Reframing clinician distress: moral injury not burnout. Fed Pract. 2019;36(9):400-2.

19. Cocker F, Joss N. Compassion fatigue among healthcare, emergency and community service workers: a systematic review. Int J Environ Res Public Health. 2016;13(6):618. https://doi.org/10. 3390/ijerph13060618.

20. Jeung DY, Kim C, Chang SJ. Emotional labor and burnout: a review of the literature. Yonsei Med J. 2018;59(2):187-93. https:// doi.org/10.3349/ymj.2018.59.2.187.

21. Kassir R. Risk of COVID-19 for patients with obesity. Obes Rev. 2020;21(6):e13034. https://doi.org/10.1111/obr.13034.

22. Rubino F, Cohen RV, Mingrone G, et al. Bariatric and metabolic surgery during and after the COVID-19 pandemic: DSS recommendations for management of surgical candidates and postoperative patients and prioritisation of access to surgery. Lancet Diabetes Endocrinol. 2020;8(7):640-8. https://doi.org/10.1016/S22138587(20)30157-1.

23. Sorensen MJ, Bessen S, Danford J, et al. Telemedicine for surgical consultations- pandemic response or here to stay? A report of public perceptions. Ann Surg. 2020; https://doi.org/10.1097/SLA. 0000000000004125.

24. McVay MA, Friedman KE, Applegate KL, et al. Patient predictors of follow-up care attendance in Roux-en-Y gastric bypass patients. Surg Obes Relat Dis. 2013;9(6):956-62. https://doi.org/10.1016/j. soard.2012.11.005.

25. Messiah SE, Sacher PM, Yudkin J, et al. Application and effectiveness of eHealth strategies for metabolic and bariatric surgery patients: a systematic review. Digital Health. 2020;6: 2055207619898987. https://doi.org/10.1177/2055207619898987.

26. Duke Clinical Research Institute. HERO: Healthcare Worker Exposure Responce and Outcomes Durham: Duke Clinical Research Institute; 2020 [cited 2020 August 28 2020]. Available from: https://heroesresearch.org/hero-hcq/.

Publisher's Note Springer Nature remains neutral with regard to jurisdictional claims in published maps and institutional affiliations. 